\title{
Clinical outcome prediction in aneurysmal subarachnoid hemorrhage - Alterations in brain- body interface
}

\section{$\operatorname{AUTHOR}(\mathrm{S}):$}

Lo, BenjaminW. Y.; Fukuda, Hitoshi; Angle, Mark; Teitelbaum, Jeanne; Macdonald, RLoch; Farrokhyar, Forough; Thabane, Lehana; Levine, MitchellA. H.

\section{CITATION:}

Lo, BenjaminW. Y....[et al]. Clinical outcome prediction in aneurysmal subarachnoid hemorrhage - Alterations in brain-body interface. Surgical Neurology International 2016, 7: S527-S537

\section{ISSUE DATE:}

2016-08-01

URL:

http://hdl.handle.net/2433/226822

\section{RIGHT:}

(C) 2016 Surgical Neurology International. This is an open access article distributed under the terms of the Creative Commons Attribution-NonCommercial-ShareAlike 3.0 License, which allows others to remix, tweak, and build upon the work non-commercially, as long as the author is credited and the new creations are licensed under the identical terms. 


\title{
Clinical outcome prediction in aneurysmal subarachnoid hemorrhage - Alterations in brain-body interface
}

\author{
Benjamin W. Y. Lo, Hitoshi Fukuda ${ }^{1}$, Mark Angle, Jeanne Teitelbaum, R. Loch Macdonald ${ }^{2}$, Forough
} Farrokhyar $^{3}$, Lehana Thabane ${ }^{4}$, Mitchell A. H. Levine ${ }^{5}$

\begin{abstract}
Department of Neurology and Neurosurgery, Montreal Neurological Institute and Hospital, McGill University, Montreal, Quebec, Canada, ${ }^{1}$ Department of Neurosurgery, Kurashiki Central Hospital, Kyoto University, Okayama, Japan, ${ }^{2}$ Division of Neurosurgery, St. Michael's Hospital, University of Toronto, Toronto, Ontario, Canada, ${ }^{3}$ Department of Surgery, Department of Clinical Epidemiology and Biostatistics, ${ }^{4}$ Department of Clinical Epidemiology and Biostatistics, ${ }^{5}$ Department of Medicine, Division of Clinical Pharmacology, Department of Clinical Epidemiology and Biostatistics, McMaster University, Hamilton, Canada

E-mail:*Benjamin W.Y. Lo - benjamin.lo@mcgill.ca; Hitoshi Fukuda - fukudaharpseal@gmail.com; Mark Angle - mark.angle@muhc.mcgill.ca; JeanneTeitelbaum - jteitelbaum@hotmail.com; R. Loch Macdonald - macdonaldlo@smh.ca; Forough Farrokhyar - farrokh@mcmaster.ca;

LehanaThabane - thabanl@mcmaster.ca; Mitchell A.H. Levine - levinem@mcmaster.ca

*Corresponding author
\end{abstract}

Received: 15 March 16 Accepted:01 June $16 \quad$ Published:0I August 16

\begin{tabular}{l} 
Abstract \\
Background: Brain-body associations are essential in influencing outcome in \\
patients with ruptured brain aneurysms. Thus far, there is scarce literature on such \\
important relationships. \\
Methods: The multicenter Tirilazad database ( 3551 patients) was used to create \\
this clinical outcome prediction model in order to elucidate significant brain-body \\
associations. Traditional binary logistic regression models were used. \\
Results: Binary logistic regression main effects model included four statistically \\
significant single prognostic variables, namely, neurological grade, age, stroke, \\
and time to surgery. Logistic regression models demonstrated the significance of \\
hypertension and liver disease in development of brain swelling, as well as the \\
negative consequences of seizures in patients with a history of myocardial infarction \\
and post-admission fever worsening neurological outcome. \\
Conclusions: Using the aforementioned results generated from binary logistic \\
regression models, we can identify potential patients who are in the high risk \\
group of neurological deterioration. Specific therapies can be tailored to prevent \\
these detriments, including treatment of hypertension, seizures, early detection \\
and treatment of myocardial infarction, and prevention of hepatic encephalopathy. \\
\begin{tabular}{l|l} 
Key Words: Aneurysmal subarachnoid hemorrhage, brain-body interactions, \\
clinical outcome prediction model
\end{tabular} \\
\hline
\end{tabular}

\section{INTRODUCTION}

The brain-body interface comprises many physiological interactions which can go awry in disease states. Under conditions of normal homeostasis, brain-body associations are frequently characterized as interactions between the human nervous system and the cardiovascular, respiratory, endocrinological, and gastrointestinal systems.
This is an open access article distributed under the terms of the Creative Commons Attribution-NonCommercial-ShareAlike 3.0 License, which allows others to remix, tweak, and build upon the work non-commercially, as long as the author is credited and the new creations are licensed under the identical terms.

For reprints contact: reprints@medknow.com

How to cite this article: Lo BW, Fukuda H,Angle M, Teitelbaum J, Macdonald $\mathrm{RL}$, Farrokhyar $\mathrm{F}$, et al. Clinical outcome prediction in aneurysmal subarachnoid hemorrhage - Alterations in brain-body interface. Surg Neurol Int 2016;7:S527-37. http://surgicalneurologyint.com/Clinical-outcome-prediction-in-aneurysmalsubarachnoid-hemorrhage---Alterations-in-brain-body-interface/ 


\section{The brain-body interface}

Cardiovascular-nervous system associations

Direct and indirect projections of the autonomic nervous system control the cardiovascular system. These projections act via the parabrachial nucleus of the midbrain and pons. The parabrachial nucleus is a relay station that transmits signals between the cerebral cortex (especially limbic cortex), amygdala, hypothalamic paraventricular nucleus, vasomotor area of the lower brainstem (including the ventrolateral medulla, medullary raphe, nucleus of the solitary tract), and the thoracolumbar interomediolateral gray column of the spinal cord. ${ }^{[2,3,4,11]}$

The autonomic nervous system mediates the heart rate (chronotrophy), rate of nervous impulse transmission through the cardiac conductive tissue (dromotropy), and force of contraction (inotropy). Blood vessel diameter and tone are also mediated via the autonomic nervous system.

Counter-regulatory feedback with the sympathetic nervous system predominates. An example of such counter-regulatory feedback is the Cushing reflex. Increase in intracranial pressure from disease states, such as intracerebral hemorrhage, leads to decreased levels of oxygen and increased local levels of hydrogen ions and carbon dioxide around the vasomotor regions of the lower brainstem secondary to anaerobic metabolism. As a counter-regulatory measure, the systemic blood pressure is elevated in an attempt to increase blood flow to the brain. With this increase in blood pressure, heart rate is reflexively decreased via the arterial baroreceptors. ${ }^{[27,28,46,48]}$

Other factors also affect the amount of autonomic nervous output from the vasomotor regions of the brainstem, including

1. Blood oxygen level,

2. Blood carbon dioxide level,

3. Pain stimulus,

4. Chemoreceptors and baroreceptors in the carotid, pulmonary, and aortic vessels sense corresponding changes,

5. Nerve impulse feedback from the lungs secondary to lung inflation and deflation, and

6. Regulatory signals between the cerebral cortex and brainstem.

\section{Respiratory-nervous system associations}

Pacemaker cells in the medulla are responsible for the autonomic control of respiration. Their rhythmic discharges are modified by pneumotaxic neurons in the pons, as well as limbic and hypothalamic lesions. ${ }^{[3,4,11]}$ Other contributions include:

1. Impulses from afferent pulmonary vagal fibers with lung inflation and deflation,

2. Spinal reflexes from cervical and thoracic spinal cord to the diaphragm and intercostal muscles,
3. Changes in the cerebrospinal fluid concentrations of carbon dioxide and hydrogen ions sensed by medullary chemoreceptors, and

4. Changes in oxygen tension sensed by baroreceptors in the aorta, cardiac atrium, and ventricle, as well as pulmonary arteries.

\section{Endocrinological-nervous system associations}

The hypothalamic-pituitary axis regulates the key aspects of homeostasis, temperature control, and endocrinological functions. This system integrates inputs from both the limbic system and brainstem in order to modulate both emotional and instinctual reactions to changes in the internal milieu. ${ }^{[2,3,11,43]}$

\section{Gastrointestinal-nervous system associations}

The gastrointestinal autonomic nervous system regulates the central nervous system inputs to the enteric system regarding gut motility and secretory functions. It works in conjunction with local paracrine pathways. ${ }^{[2,3,11]}$

Aneurysmal subarachnoid hemorrhage and alterations in brain-body associations

Patients with ruptured brain aneurysms and associated subarachnoid hemorrhage (SAH) have a mortality rate of at least $45 \%$ in the first month after rupture. ${ }^{[32,33]}$ Neurological damage can be from the primary injury of the aneurysm rupture itself and a number of secondary injurious processes that can further worsen the affected individual's neurological state. These secondary processes can be related to the nervous system, such as rebleeding from the ruptured aneurysm, brain swelling, occurrence of a delayed second stroke, brain blood vessel vasospasm leading to strokes, seizures, and over accumulation of brain spinal fluid causing hydrocephalus. In addition, other body organ systems can be affected, such as myocardial infarction and over accumulation of fluid in lungs causing pulmonary edema. Together, these processes can lead to long-term disability. Types of disability include physical, neurocognitive, and psychological impairment. Table 1 summarizes alterations in physiological interactions in the brain-body interface.

\section{The Tirilazad database}

Tirilazad was a 21-aminosteroid compound produced by Pharmacia and Upjohn, Kalamazoo, Michigan. It was originally investigated as a free radical scavenger for the potential treatment of cerebral vasospasm. Between 1991 and 1997, 3551 patients from 171 centers in 22 countries in North America, Europe, Australia, New Zealand, and Africa participated in five randomized, double-blind, placebo-controlled trials of Tirilazad. ${ }^{[12,13,21,25,26]}$

Included patients were adults with evidence of aneurysmal SAH. The patients received either placebo or active agent (in intravenous doses of $0.6,2.6$, or $15 \mathrm{mg} / \mathrm{kg} / \mathrm{day}$ depending on the trial) from the third to tenth day after onset of SAH. Excluded patients were: 
Table 1: Brain-body interface under normal homeostasis and after aneurysmal subarachnoid hemorrhage

\begin{tabular}{l} 
Brain-body interface under normal homeostasis \\
\hline Cardiovascular system \\
-Autonomic nervous control of cardiovascular system: \\
1. Counter-regulatory feedback with sympathetic nervous \\
system, and \\
2. Primary relay station being vasomotor regions of the \\
lower brainstem \\
Pulmonary system \\
-Automatic control of respiration by lower brainstem's \\
pacemaker cells with additional modifications by: \\
1. Brainstem chemoreceptors and vascular \\
baroreceptors, \\
2. Brain (limbic, hypothalamic, and brainstem) inputs and \\
spinal (cervical and thoracic cord) reflexes, and \\
3. Pulmonary local volume and vascular controls \\
Endocrine system \\
-Hypothalamic-pituitary axis coordinates key aspects of \\
homeostasis \\
1. Counter-regulatory feedback with target end organs \\
including the adrenal glands
\end{tabular}

1. Those with traumatic SAH,

2. Those with infectious mycotic aneurysms,

3. Severe underlying medical illnesses including serious cardiovascular diseases, such as myocardial infarction within the previous 6 months, uncontrolled hypertension, serious cardiac arrthymias, or congestive heart failure,

4. Pregnant or lactating patients,

5. Patients taking corticosteroids or calcium channel blockers other than nimodipine,

6. Known intolerance to calcium channel blockers, and

7. Patients whose aneurysms were treated with Gulielmi or other detachable coils.

Patients in the placebo and treatment arms were otherwise treated similarly. Over $85 \%$ of patients underwent surgical clipping, and half of them were operated within the first 48 hours. Baseline demographics in both the arms were balanced in terms of sex, age, number of pre-existing medical conditions, mean time to treatment, mean admission systolic blood pressure, admission neurological grade, ruptured aneurysm location, amount of blood visualized on admission computed tomography (CT) scan (measured by the Fisher scale taking into account thickness of the blood clot with or without intraventricular component). The potential confounder of patient sex was accounted for in statistical analysis in the North American Tirilazad trial where fewer female patients were randomized to the treatment arm. ${ }^{[13]}$ The percentages of patients experiencing neurological and systemic disabilities were similar in different treatment groups. ${ }^{[12,13,21,25,26]}$

Only $1 \%$ of patients were lost to follow-up. The primary outcome measure was the patient's Glasgow Outcome Score (GOS) at 3 months after aneurysmal rupture. GOS is a 5-point scale, defined as: 5 - good recovery with normal life activities despite minor deficits, 4 - moderate disability being disabled but independent, 3 - severe disability being conscious but dependent, 2 - persistent vegetative state, and 1 - death. ${ }^{[19]}$

Two systematic reviews involving the five Tirilazad trials found no substantial heterogeneity among these trials, and no significant differences between treatment and placebo arms, or among patients who were administered different dosages of Tirilazad regarding the number of patients who died, the number of adverse events, or neurological outcome at 3 months follow-up. ${ }^{[17,49]}$

\section{Objectives}

Using data from the placebo and Tirilazad arms of the five clinical trials, we aim to create a clinical outcome prediction model using binary logistic regression and investigate the potential factors for outcome prediction in patients with ruptured brain aneurysms. In particular, we assess the interaction of potential factors and explore the role for possible associations between the brain and other organ systems.

\section{MATERIALS AND METHODS}

The Tirilazad database was used to investigate the potential factors for outcome prediction in aneurysmal $\mathrm{SAH}$ and to assess interactions of factors between the brain and other organ systems.

Table 2 summarizes the independent variables recorded in the Tirilazad database.

The dependent variable used for statistical analysis is the dichotomized GOS at three months, that is, good 
SNI: Neurovascular 2016,Vol 7: Suppl 18 - A Supplement to Surgical Neurology International

Table 2: Independent variables recorded in the Tirilazad database

\begin{tabular}{|c|c|c|}
\hline Demographic sactors & Neurological factors & Systemic factors \\
\hline 1. Sex & Non-treatment related factors & \\
\hline 2. Age (years) & 1. Hospital admission neurological grade (Hunt and Hess) & 1. Systolic blood pressure $(\mathrm{mmHg})$ \\
\hline \multirow[t]{22}{*}{ 3. Patient's weight (kg) } & 1 - Asymptomatic & 2. Diastolic blood pressure $(\mathrm{mmHg})$ \\
\hline & 2 - Headaches, cranial nerve palsy & 3. Temperature on admission (degree Celsius) \\
\hline & 3 - Mild focal deficit, confusion & 4. Occurrence of fever one week after hospital admission \\
\hline & 4 - Stupor, moderate to severe hemiparesis & 5. History of hypertension \\
\hline & 5 - Deep coma, moribund & 6. History of angina \\
\hline & 2. Aneurysm size ( $\leq 12 \mathrm{~mm}$ or $>12 \mathrm{~mm}$ ) & 7. History of myocardial infarction \\
\hline & 3. Presence of angiographic vasospasm on hospital admission & 8. History of diabetes mellitus \\
\hline & 4. Presence of intraventricular hemorrhage & 9. History of hepatic disease \\
\hline & 5. Presence of intracerebral hemorrhage & 10. History of thyroid disease \\
\hline & 6. Location of aneurysm (anterior or posterior circulation) & 11. Development of pulmonary edema \\
\hline & 7. Thickness of subarachnoid hemorrhage ( $\leq 1 \mathrm{~mm}$ or $>1 \mathrm{~mm}$ ) & \\
\hline & 8. Prior episode of subarachnoid hemorrhage & \\
\hline & 9. History of migraines & \\
\hline & 10. Presence of hydrocephalus & \\
\hline & 11. Development of cerebral edema & \\
\hline & 12. Occurrence of stroke post admission & \\
\hline & 13. Development of vasospasm during course of treatment & \\
\hline & 14. Seizures requiring antiepileptic medications & \\
\hline & Treatment related factors & \\
\hline & 1. Time to surgical treatment (hours) & \\
\hline & 2. Treatment arm & \\
\hline & 3. Severe vasospasm requiring balloon angioplasty & \\
\hline
\end{tabular}

or poor outcome. Good outcome represents functional independence (GOS 5 or 4). Poor outcome represents functional dependence (GOS 3), persistent vegetative state (GOS 2) or death (GOS 1). ${ }^{[16]}$

Table 3 summarizes neurologic and systemic factors, as well as a priori hypotheses investigated possible interactions between brain and other organ systems.

Using IBM's the Statistical Package for the Social Sciences version $19.0^{\mathrm{TM}}$, independent variables were entered into univariate models in which Chi-square analyses were performed to investigate associations with poor outcome. Two-way interaction terms that included neurologic and systemic prognostic factors were examined [Table 3]. Variables that reached a probability of 0.10 were then entered into a multivariable binary logistic regression model. Variables that reached $P \leq 0.05$ were deemed significant. For Hosmer and Lemeshow test for goodness of fit of the model, $P \geq 0.05$ was deemed significant. ${ }^{[15,44]}$ Multivariable analyses had at least 10 participants per independent variable, which is the minimum number required for a stable multivariable model using logistic regression. ${ }^{[15,44]}$

In the multivariable logistic regression model, the following diagnostics were performed:
1. Correlation matrix to rule out multicollinearity among predictor variables,

2. Hosmer and Lemeshow test to ensure good data fit to model and model calibration,

3. C statistic and classification to examine model discrimination, and

4. Split-sample analysis (70\% training and 30\% testing) to examine model generalizability.

\section{RESULTS}

Patient selection and participation in the Tirilazad trials are summarized in Figure 1. Characteristics of the study patient population are summarized in Table 4.

Correlated matrix of the included independent variables was examined to ensure that there were no correlated variables. Variables and interaction terms that reached a probability of 0.10 were entered into the multivariable binary logistic regression model. These variables and interaction terms are summarized in Table 5. Significant single prognostic variables and interaction terms were entered into a multivariable binary logistic regression model. The final model includes significant single prognostic variables and interaction terms summarized in Table 6. 


\section{Table 3: A priori hypotheses for brain-body interactions and their rationale}

\section{Systemic factors and rationale for investigating brain-body interactions}

Age

Age, along with clinical pre-operative grade and CT findings are associated with poor neurological outcome in aneurysmal SAH. ${ }^{[14,42]}$ Both cerebral and systemic atherosclerotic changes occur as one ages

hypertension

Hypertension is associated with poor neurological outcome in aneurysmal SAH as it predisposes to hemodynamic related structural damage in cerebral blood vessels, including endothelial damage and decreased elasticity of blood vessel wall. ${ }^{[1,29,30]}$

History of diabetes

Diabetes and poor glycemic control are associated with symptomatic vasospasm. ${ }^{[10]}$ Insulin resistance is associated with dysfunction of vascular resistance vessels, including endothelial hypertrophy and altered contractile properties.

History of liver disease

Hepatic dysfunction is associated with poor clinical grade at hospital admission. During the course of treatment, liver dysfunction is observed in up to $25 \%$ of aneurysmal SAH patients. ${ }^{[4]}$

History of thyroid disease

Sick euthyroid syndrome may be observed in aneurysmal SAH patients with previously normal functioning thyroid gland. During acute physiological stress, these patients may have decreased amounts of thyroid hormone, and inhibition of thyroxine production by elevated cortisol levels. ${ }^{[7,34]}$

Occurrence of fever at 1 week post admission

Fevers are associated with increased cerebral metabolic rates. Even one episode of fever after aneurysmal SAH can be associated with poor outcome. ${ }^{[45]}$

History of myocardial infarction

Patients with a history of myocardial infarction are prone to troponin elevations and decompensated heart failure after SAH. ${ }^{[22]}$ Those with elevated troponin levels and heart failure are more likely to have significant morbidity and mortality

Neurologic factors and specific a priori hypotheses

Neurological grade

I will investigate the degree by which admission neurological grade influences clinical outcome if the aneurysmal SAH patients also have the following conditions, as they are prone to complications arising from them:

(1) Increasing age (age and neurological grade)

(2) Hypertension (hypertension and neurological grade)

(3) Diabetes (diabetes and neurological grade)

(4) Liver disease (liver disease and neurological grade)

(5) Thyroid disease (thyroid disease and neurological grade)

(6) Fever (fever and neurological grade), and

(7) Myocardial infarction (myocardial infarction and neurological grade)

Occurrence of stroke post admission

I hypothesize that aneurysmal SAH patients who experience post admission stroke may have poor clinical outcome if they also have the following conditions:

(1) Increasing age (age and stroke)

(2) Hypertension (hypertension and stroke)

(3) Diabetes (diabetes and stroke)

(4) Liver disease (liver disease and stroke)

(5) Fever (fever and stroke), and

(6) Myocardial infarction (myocardial infarction and stroke)

Aneurysmal SAH patients with post admission stroke and the aforementioned systemic conditions are predisposed to related complications including rebleeding, seizures, cerebral edema, delayed stroke, and vasospasm

Development of vasospasm during course of treatment

Aneurysmal SAH patients who experience vasospasm may be predisposed to poor clinical outcome as they are more likely to experience complications including delayed stroke and rebleeding if they concomitantly have the following conditions:

(1) Hypertension (hypertension and vasospasm)

(2) Diabetes (diabetes and vasospasm)

(3) Liver disease (liver disease and vasospasm)

(4) Fever (fever and vasospasm), and

(5) Myocardial infarction (myocardial infarction and vasospasm)

Seizures requiring antiepileptic medications 
Table 3: Contd...

I hypothesize that aneurysmal SAH patients with the following systemic conditions who experience seizures may be predisposed to poor clinical outcome arising from complications including status epilepticus, cerebral edema, vasospasm, and delayed stroke:

(1) Increasing age (age and seizures)

(2) Hypertension (hypertension and seizures)

(3) Diabetes (diabetes and seizures)

(4) Liver disease (liver disease and seizures)

(5) Fever (fever and seizures), and

(6) Myocardial infarction (myocardial infarction and seizures)

Aneurysm size

Aneurysmal SAH patients with hypertension (hypertension and aneurysm size) and myocardial infarction (myocardial infarction and aneurysm size) may experience rebleeding complicating their clinical course leading to poor clinical outcome.

Presence of intraventricular hemorrhage

I hypothesize that aneurysmal SAH patients with intraventricular hemorrhage and the following systemic conditions are more likely to experience complications including hydrocephalus and rebleeding which may lead to poor clinical outcome:

(1) Increasing age (age and intraventricular hemorrhage)

(2) Hypertension (hypertension and intraventricular hemorrhage)

(3) Diabetes (diabetes and intraventricular hemorrhage)

(4) Liver disease (liver disease and intraventricular hemorrhage)

(5) Fever (fever and intraventricular hemorrhage), and

(6) Myocardial infarction (myocardial infarction and intraventricular hemorrhage)

Presence of intracerebral hemorrhage

Aneurysmal SAH patients with post admission intracerebral hemorrhage are prone to poor clinical outcome arising from complications including rebleeding, seizures, cerebral edema, and vasospasm, if they also have the following conditions:

(1) Increasing age (age and intracerebral hemorrhage)

(2) Hypertension (hypertension and intracerebral hemorrhage)

(3) Diabetes (diabetes and intracerebral hemorrhage)

(4) Liver disease (liver disease and intracerebral hemorrhage)

(5) Fever (fever and intracerebral hemorrhage), and

(6) Myocardial infarction (myocardial infarction and intracerebral hemorrhage)

Development of cerebral edema

I hypothesize that aneurysmal SAH patients who develop post rupture cerebral edema and, concomitantly, have the following conditions, may be prone to poor clinical outcome:

(1) Increasing age (age and cerebral edema)

(2) Hypertension (hypertension and cerebral edema)

(3) Diabetes (diabetes and cerebral edema)

(4) Liver disease (liver disease and cerebral edema)

(5) Fever (fever and cerebral edema), and

(6) Myocardial infarction (myocardial infarction and cerebral edema)

These patients may experience seizures and delayed stroke as possible related complications

The multivariable binary logistic regression model found four statistically significant single prognostic variables, namely, admission neurological grade, age, time to surgery and post-admission stroke, and four significant interaction terms. These observations are listed as follows:

1. The odds of poor outcome associated with one unit increase in neurological grade is increased by a factor of 2.06,

2. The odds of poor outcome associated with one unit (every 5 years) increase in age is increased by a factor of 1.28 ,
3. The odds of poor outcome associated with one unit (every hour) increase in time to surgery is increased by a factor of 1.01 , and

4. The odds of poor outcome is increased by a factor of 4.0 when the aneurysmal SAH patient experiences post-hospital admission stroke.

The multivariable binary logistic regression model also found four statistically significant interaction terms. These observations are listed as follows:

1. The odds of poor outcome is increased by a factor of 2.39 when the aneurysmal SAH patient experiences fever 1 week post-hospital admission and seizures, 
SNI: Neurovascular 2016,Vol 7: Suppl I8 - A Supplement to Surgical Neurology International

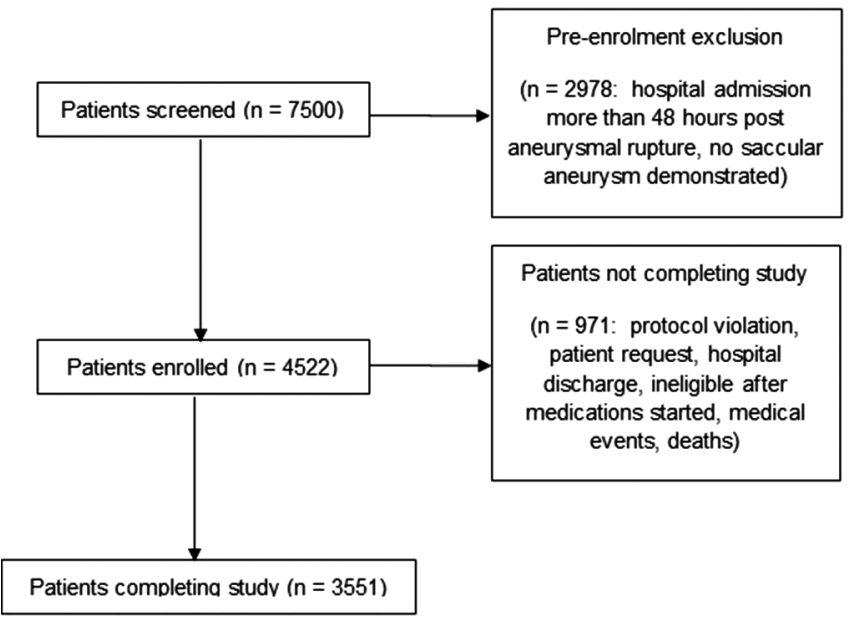

Figure I: Patient selection and participation in the Tirilazad trials

2. The odds of poor outcome is increased by a factor of 5.47 when the aneurysmal SAH patient with history of hepatic disease develops cerebral edema,

3. The odds of poor outcome is increased by a factor of 2.66 when the aneurysmal SAH patient with history of hypertension develops cerebral edema, and

4. The odds of poor outcome is increased by a factor of 3.05 when the aneurysmal SAH patient with history of myocardial infarction develops seizures.

The Omnibus Tests of Model Coefficients indicated that this model was statistically significant $($ Chi-square $=887.90, P<0.01)$. This model showed a large significant reduction in -2 log likelihood (-2 LL from 2240 to $1699, P<0.01)$. Nagelkerke $R^{2}$ statistic indicated that approximately $47 \%$ of the variance in poor neurologic outcome could be predicted from the combination of the significant terms. In terms of model discrimination [Figure 2], the c statistic was $0.87(95 \%$ confidence interval 0.86-0.89).

Classification table indicated that $84 \%$ of subject outcomes were predicted correctly. In terms of model calibration, the Hosmer and Lemeshow test showed a Chi-square of 11.38, $P=0.18$, suggesting that the model appeared to fit the data well. Generalizability of model findings was supported by split sample validation $(70 \%$ training and 30\% testing). The following are the findings:

1. No multicollinearity (standard errors for beta coefficients less than 2),

2. Individual relationship Wald statistic significance less than alpha level of 0.05 , and

3. Classification accuracy rate for training sample was $84.5 \%$, whereas classification accuracy rate for testing sample was $82.5 \%$, both satisfying minimum requirement for holdout sample $(0.9 \times 84.5 \%=76 \%)$, and

4. Classification accuracy (84.5\%) was 1.025 times chance accuracy $(82.5 \%)$.
Table 4: Characteristics of the study patient population

\begin{tabular}{|c|c|}
\hline Characteristic $(n=3551)$ & Statistic \\
\hline \multicolumn{2}{|l|}{ Demographic factors } \\
\hline Mean age (years) & $51.7 \pm 13.2$ \\
\hline Female sex (percent) & $2933(82.6)$ \\
\hline Patient weight (kilogram) & $69.5 \pm 15.0$ \\
\hline \multicolumn{2}{|l|}{ Neurological factors } \\
\hline \multicolumn{2}{|l|}{ Non-treatment related } \\
\hline \multicolumn{2}{|l|}{ Hospital admission neurological grade } \\
\hline Hunt and Hess Grade $1(\%)$ & $1289(36.3)$ \\
\hline Hunt and Hess Grade $2(\%)$ & $1045(29.4)$ \\
\hline Hunt and Hess Grade $3(\%)$ & $417(11.7)$ \\
\hline Hunt and Hess Grade $4(\%)$ & $355(10.0)$ \\
\hline Hunt and Hess Grade 5 (\%) & $445(12.5)$ \\
\hline Aneurysm size $\leq 12 \mathrm{~mm}(\%)$ & $2594(73.0)$ \\
\hline Presence of admission angiographic vasospasm (\%) & $412(11.6)$ \\
\hline Presence of intraventricular hemorrhage (\%) & $1588(44.7)$ \\
\hline Presence of intracerebral hematoma (\%) & $818(23.0)$ \\
\hline Posterior circulation location of aneurysm (\%) & $498(14.0)$ \\
\hline Subarachnoid hemorrhage thickness $\leq 1 \mathrm{~mm}(\%)$ & $1214(34.2)$ \\
\hline Prior episode of SAH (\%) & $340(9.6)$ \\
\hline History of migraines (\%) & $915(25.8)$ \\
\hline Presence of hydrocephalus (\%) & $1497(42.2)$ \\
\hline Development of cerebral edema (\%) & $381(10.7)$ \\
\hline Occurrence of post admission stroke (\%) & $979(27.6)$ \\
\hline Development of vasospasm during treatment (\%) & $1059(29.8)$ \\
\hline \multicolumn{2}{|l|}{ Treatment related } \\
\hline Seizures requiring antiepileptic medications (\%) & $2313(65.1)$ \\
\hline Mean time to surgical treatment (hours) & $0.9 \pm 1.9$ \\
\hline Treatment arm receiving Tirilazad (\%) & $2173(61.2)$ \\
\hline Severe vasospasm needing balloon angioplasty (\%) & $158(4.4)$ \\
\hline \multicolumn{2}{|l|}{ Systemic factors } \\
\hline Mean systolic blood pressure (mmHg) & $141.2 \pm 24.7$ \\
\hline Mean diastolic blood pressure (mmHg) & $76.6 \pm 15.2$ \\
\hline Mean temperature on admission (degree Celsius) & $37.0 \pm 0.9$ \\
\hline Occurrence of fever one week after admission (\%) & $1753(49.4)$ \\
\hline History of hypertension (\%) & $1217(34.3)$ \\
\hline History of angina (\%) & $129(3.6)$ \\
\hline History of myocardial infarction (\%) & $87(2.5)$ \\
\hline History of diabetes mellitus (\%) & $155(4.4)$ \\
\hline History of hepatic disease (\%) & $127(3.6)$ \\
\hline History of thyroid disease (\%) & $291(8.2)$ \\
\hline Development of pulmonary edema (\%) & $313(8.8)$ \\
\hline
\end{tabular}

\section{DISCUSSION}

There has been scarce literature attempting to characterize complex brain-body associations in patients with ruptured brain aneurysms. This analysis included both non-treatment and treatment related prognostic factors, as well as two-way interactions, which described clinically relevant brain-body associations in aneurysmal SAH. 
SNI: Neurovascular 2016,Vol 7: Suppl I8 - A Supplement to Surgical Neurology International

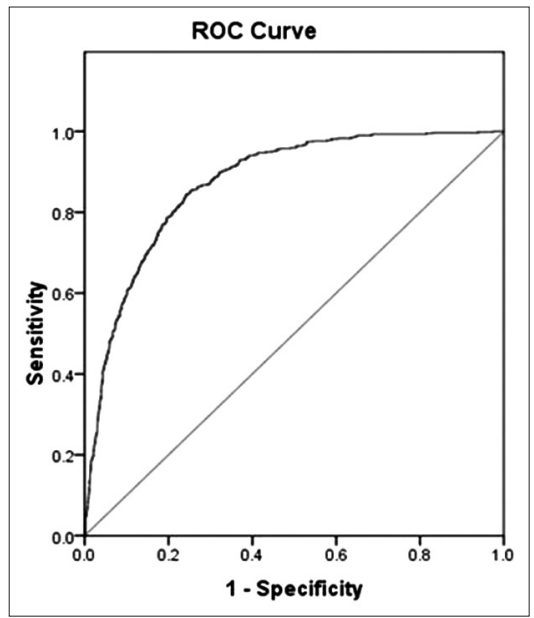

Figure 2: ROC curve to analyze logistic regression model discrimination. Output figure generated by IBM SPSS Version 19.0 (Armonk NY)

The main effects logistic regression model confirmed the significance of neurological grade, age, stroke, and time to surgery in outcome prognosis in aneurysmal SAH.

This study also demonstrates that the odds of poor neurological outcome is increased by a factor of 4 in aneurysmal SAH patients who develop post-admission strokes (OR: 4.03, 95\% CI: 2.11-7.69, P < 0.01). With modern modalities of routine cerebral surveillance imaging over the course of treatment, up to half of aneurysmal SAH patients have observed non-resolving hypodensities and persistent areas of cerebral perfusion deficits, which is consistent with stroke. It is increasingly recognized that cerebral infarction after aneurysmal SAH may occur early after aneurysmal rupture or in a delayed manner. Factors associated with development of cerebral infarction include admission neurological status, treatment related complications, and occurrence of symptomatic vasospasm. ${ }^{[23,41]}$ Secondary injury cascade events predisposing aneurysmal SAH patients to post admissions strokes include: (1) Microthrombi formation, (2) cortical spreading depression, (3) microvascular constriction, (4) proliferation of pro-inflammatory cascade, (5) presence of blood-brain barrier disruption, and (6) inadequate collateral circulations.

In addition, this study found that timing to surgical treatment of ruptured aneurysms is significant in determining neurological outcome. This is supported from the observation that shorter time interval to treatment is associated with reduction in aneurysmal rebleeding rates. ${ }^{[38]}$

We also make the observation that development of cerebral edema, in the context of history of hypertension and liver disease, has significant impact on neurologic outcome deterioration in aneurysmal SAH.
Table 5: Univariate analysis main effects variables and interaction terms

\begin{tabular}{|c|c|c|c|}
\hline Variable terms & $\begin{array}{l}\text { Odds } \\
\text { ratios }\end{array}$ & $\begin{array}{l}\text { 95\% confidence } \\
\text { intervals }\end{array}$ & $P$ value \\
\hline \multicolumn{4}{|l|}{ Main effects variables } \\
\hline Neurological grade & 2.06 & $1.82-2.31$ & $<0.01$ \\
\hline Age (years) & 1.05 & $1.04-1.07$ & $<0.01$ \\
\hline Time to surgery (hours) & 1.00 & $1.00-1.01$ & 0.03 \\
\hline Fever on day 8 & 1.94 & $1.51-2.49$ & $<0.01$ \\
\hline Aneurysm size & 1.37 & $1.04-1.80$ & 0.02 \\
\hline Admission vasospasm & 1.54 & $1.04-2.30$ & 0.03 \\
\hline Intraventricular hemorrhage & 1.33 & $1.02-1.73$ & 0.04 \\
\hline Brain Edema & 1.84 & $1.29-2.62$ & $<0.01$ \\
\hline Intracerebral hemorrhage & 1.39 & $1.04-1.87$ & 0.03 \\
\hline History of hypertension & 1.37 & $1.05-1.79$ & 0.02 \\
\hline History of myocardial infarction & 2.07 & $1.01-4.27$ & 0.06 \\
\hline Post admission stroke & 6.35 & $4.90-8.24$ & $<0.01$ \\
\hline Previous subarachnoid hemorrhage & 1.60 & $1.07-2.39$ & 0.02 \\
\hline Aneurysm location & 1.72 & $1.18-2.51$ & 0.01 \\
\hline Vasospasm & 1.58 & $1.21-2.06$ & $<0.01$ \\
\hline Seizures & 1.68 & $1.28-2.21$ & $<0.01$ \\
\hline \multicolumn{4}{|l|}{ Interaction terms } \\
\hline Fever on day 8 by aneurysm size & 2.63 & $2.10-3.31$ & $<0.01$ \\
\hline $\begin{array}{l}\text { Fever on day } 8 \text { by intraventricular } \\
\text { hemorrhage }\end{array}$ & 3.21 & $2.68-3.83$ & $<0.01$ \\
\hline Fever on day 8 by seizures & 2.66 & $2.24-3.14$ & $<0.01$ \\
\hline Brain edema by hepatic disease & 3.50 & $1.24-9.86$ & 0.02 \\
\hline $\begin{array}{l}\text { Brain edema by history of } \\
\text { hypertension }\end{array}$ & 4.18 & $2.96-5.91$ & $<0.01$ \\
\hline Hydrocephalus by hepatic disease & 1.82 & $1.02-3.23$ & 0.04 \\
\hline $\begin{array}{l}\text { History of myocardial infarction } \\
\text { by subarachnoid hemorrhage } \\
\text { thickness }\end{array}$ & 2.63 & $1.53-4.53$ & $<0.01$ \\
\hline $\begin{array}{l}\text { History of myocardial infarction } \\
\text { by seizures }\end{array}$ & 3.35 & $1.86-6.05$ & $<0.01$ \\
\hline
\end{tabular}

Table 6: Statistically significant terms in the final binary logistic regression model

\begin{tabular}{lccc}
\hline Variable Terms & $\begin{array}{c}\text { Odds } \\
\text { ratios }\end{array}$ & $\begin{array}{c}\text { 95\% confidence } \\
\text { intervals }\end{array}$ & $\boldsymbol{P}$ value \\
\hline Neurological grade & 2.06 & $1.83-2.32$ & $<0.01$ \\
Age (per year) (for every $\Delta 5$ years) & 1.06 & $1.05-1.07$ & $<0.01$ \\
& {$[1.28]$} & {$[1.22-1.42]$} & \\
Time to surgery (hour) & 1.01 & $1.00-1.02$ & 0.02 \\
Stroke & 4.03 & $2.11-7.69$ & $<0.01$ \\
Seizures by fever on day 8 & 2.39 & $1.86-3.06$ & $<0.01$ \\
Brain edema by hepatic disease & 5.47 & $1.13-26.46$ & 0.03 \\
Brain edema by hypertension & 2.66 & $1.59-4.45$ & $<0.01$ \\
Seizures by myocardial infarction & 3.05 & $1.35-6.87$ & 0.01
\end{tabular}

By itself, development of cerebral edema may predispose the aneurysmal SAH to poor neurological outcome (OR: 1.84, 95\% CI: 1.29-2.62, $P<0.01$, univariate analysis). 
Further examination of systemic factors revealed that aneurysmal SAH patients with a history of hypertension and development of cerebral edema have 2.7 fold increased odds of poor neurologic outcome (OR: 2.66, 95\% CI $1.59-4.45, P<0.01)$. Patients with a history of hypertension are more prone to defective cerebral autoregulation. When cerebral dysautoregulation is present after aneurysmal $\mathrm{SAH}$, brain engorgement can occur as plasma proteins leak from capillaries with increased permeability. Extracellular vasogenic edema may follow as a result of increased hydrostatic pressures, with predilection for posterior cerebral circulation territories. ${ }^{[39,40]}$ It is important, therefore, to recognize that chronic hypertensive patients have altered elevated blood pressure ranges for autoregulation. It is also essential to monitor the development of cerebral edema in these patients.

This study also makes the novel observation that development of cerebral edema in aneurysmal SAH patients with a history of liver dysfunction markedly increases likelihood for poor outcome (OR: 5.47, 95\% CI: $1.13-26.46, P=0.03)$. Similar to patients with hypertensive history, patients with chronic liver disease have been shown to have altered cerebral autoregulation and cerebral blood flow with decreased cerebral blood flow in the anterior cingulum and increased blood flow in the basal ganglia and occipital lobes at baseline. In acute states of ruptured cerebral aneurysms, these patients' blood brain barriers become disrupted with marked increased cerebral blood flow secondary to luxuriant perfusion, thus, predisposing them to the development of vasogenic edema. In addition, cytotoxic osmoregulatory mechanisms are involved whereby astrocytes swell secondary to the toxic effects of ammonia and glutamate. The end result is a vicious cycle of neuronal swelling and death, marked increased in cerebral blood flow (cerebral hyperemia), and cerebral edema. ${ }^{[5,6,8,37]}$ It is important, therefore, to prevent the development of hepatic encephalopathy and monitor for cerebral edema in aneurysmal SAH patients who have chronic liver dysfunction.

Seizures post-aneurysmal SAH increase mortality. The observed incidence of seizures can be as high as $15 \%$ in the aneurysmal SAH patient population. ${ }^{[45,47]}$ In this study, we observed that seizures increase the odds of poor outcome by a factor of 1.68 (95\% CI: 1.28-2.21, P < 0.01, univariate analysis). However, occurrence of seizures in the clinical settings of post-admission fever and background history of myocardial infarction significantly increases morbidity and mortality. The epileptic aneurysmal SAH patient who develops post-admission fever is predisposed to poor outcome (OR: 2.39, 95\% CI: 1.86-3.06, $P<0.01$ ). Fevers increase cerebral metabolic rate and can exacerbate secondary injury. Early onset fevers can be secondary to dysfunction of temperature regulation centers in the hypothalamus whereas late onset fevers are more likely to be infectious, but can include fevers secondary to drugs and pulmonary embolism. ${ }^{[27,28,36,48]}$ Exogenous and endogenous pyrogens increase the propensity for fevers and seizure development. In febrile states, inflammatory cytokines increase neuronal excitability via temperature sensitive ion channels leading to an increased likelihood of synchronized neuronal activity. ${ }^{[0,20,34,35]}$ Not only is it essential to monitor and treat both seizures and fevers themselves, it is also important to search for underlying etiologies, including infections, pulmonary embolism, drug-drug interactions and delayed strokes which may alter seizure thresholds in the febrile aneurysmal SAH patient.

Finally, our analysis makes the observation that seizures in the setting of history of myocardial infarction increase the odds of poor outcome by a factor of 3.05 (95\% CI: $1.35-6.87, P=0.01)$. Repetitive autonomic stimulation can occur in the actively seizing aneurysmal SAH patient in a lock step phenomenon, which can trigger the development of cardiac ictal arrthymias. ${ }^{[6,18,29]}$ Continuous cardiac sympathetic discharges and cortical epileptiform activity can occur in a synchronized time-locked manner. These repetitive synchronized autonomic sympathetic discharges lead to cardiac ischemia and structural damage to the myocardium. ${ }^{[24]}$ In aneurysmal SAH, patients with pre-existing coronary artery disease and seizures, the propensity of cardiac ischemia is increased, along with potentially fatal multi-systemic complications, including development of neurogenic pulmonary edema and respiratory suppression associated with fatal cardiac tachy or bradyarrthymias, or cardiac asystole. Multi-system critical care cardiovascular and respiratory supports, therefore, are essential in these epileptic aneurysmal SAH patients in order to maximize their chances of survival.

\section{Methodological strengths}

Our analyses made use of a large multicenter database of aneurysmal SAH patients. Multivariable logistic regression model is a robust statistical technique applicable to this patient cohort because there were: (1) No significant differences in patient outcome (benefit or harm) between placebo and treatment arms, (2) no substantial heterogeneity among different Tirilazad trials, (3) baseline demographics in both arms were balanced, (4) patients in both arms were treated similarly, with statistical analyses accounting for potential confounders, (5) similar percentages of patients experiencing neurological and systemic disabilities in placebo and different treatment groups, and (6) no significant between-center and between-country effects on patient outcome. ${ }^{[12,13,17,21,25,26,31,49]}$

In addition, we investigated both single prognostic factors and explored brain-body interactions. In so doing, several novel observations were found which significantly influence clinical outcome in aneurysmal SAH patients. 
Furthermore, we examined model discrimination, calibration and validity.

\section{Limitations}

This is a retrospective observational study which included heterogeneous clusters of patients from different geographical regions. Patients of various genetic backgrounds may exhibit different clinical responses to treatments such as pharmacokinetic profiles to medications. In addition, the Tirilazad database did not capture important modifiable variables of behaviours including smoking and alcohol consumption. Referral bias is also present because these patients were admitted to and treated at specialized centers with neurosurgical and critical care capabilities.

\section{Future directions}

Further studies can be carried out to generalize these observations to other aneurysmal SAH patient populations, including those undergoing coiling. ${ }^{[32]}$ Further delineation can also be carried out to investigate the impact of brain interactions with other body systems, such as renal system, on neurological outcome in aneurysmal SAH patients. In addition, clinical prediction models formulated using multivariable logistic regression analysis and other techniques, such as classification and regression tree analysis, can be adopted as clinical prognostication tools, openly accessible to the clinician in online downloadable formats and applications.

\section{Financial support and sponsorship}

Nil.

\section{Conflicts of interest}

There are no conflicts of interest.

\section{REFERENCES}

I. Andreasen TH, Bartek J Jr, Andresen M, Springborg JB, Romner B. Modifiable risk factors for aneurysmal subarachnoid hemorrhage. Stroke 2013;44:3607-12.

2. Barrett K, Ganong W. Ganong's review of medical physiology. New York: McGraw-Hill Medical; 2010.

3. Bederson J, AANS Publications Committee. Subarachnoid hemorrhage: Pathophysiology and management. Park Ridge, III.: American Association of Neurological Surgeons; 1997.

4. Butterworth R. The liver-brain axis in liver failure: Neuroinflammation and encephalopathy. Nat Rev Gastroenterol Hepatol 2013;10:522-8.

5. Devinsky $\mathrm{O}$. Effects of seizures on autonomic and cardiovascular function. Epilepsy Curr 2004;4:43-6.

6. Detry O, De Roover A, Honore P, Meurisse M. Brain edema and intracranial hypertension in fulminant hepatic failure: Pathophysiology and management. World J Gastroenterol 2006; 12:7405-12.

7. Dimopoulou I, Kouyialis AT, Tzanella M, Armaganidis A, Thalassinos N, Sakas DE, et al. High incidence of neuroendocrine dysfunction in long-term survivors of aneurysmal subarachnoid hemorrhage. Stroke 2004;35:2884-9.

8. Dooley J, Sherlock S. Sherlock's diseases of the liver and biliary system. Chichester, West Sussex; Hoboken, NJ: Wiley-Blackwell; $201 \mathrm{I}$.

9. Dube CM, Brewster AL, Baram TZ. Febrile seizures: Mechanisms and relationship to epilepsy. Brain and Dev 2009;31:366-7I.

10. Dumont T, Rughani A, Silver J, Tranmer Bl. Diabetes mellitus increases risk of vasospasm following aneurysmal subarachnoid hemorrhage independent of glycemic control. Neurocrit Care. 2009; I 1:183-9.

II. Guyton A, Hall J. Textbook of medical physiology. Philadelphia: Saunders; 2000.

12. Haley EC Jr, Kassell NF, Alves WM, Weir BK, Hansen CA. Phase II trial of tirilazad in aneurysmal subarachnoid hemorrhage. A report of the Cooperative Aneurysm Study. J Neurosurg 1995;82:786-90.

13. Haley EC Jr, Kassell NF, Apperson-Hansen C, Maile MH, Alves WM. A randomized, double-blind, vehicle-controlled trial of tirilazad mesylate in patients with aneurysmal subarachnoid hemorrhage: A cooperative study in North America. J Neurosurg. 1997;86:467-74.

14. Horiuchi T, Tanaka Y, Hongo K. Surgical treatment for aneurysmal subarachnoid hemorrhage in the $8^{\text {th }}$ and $9^{\text {th }}$ decades of life. Neurosurgery 2005;56:469-75.

15. Hosmer D, Lemeshow S, Sturdivant R. Applied logistic regression. Hoboken, $\mathrm{NH}$ : Wiley; 2013.

16. Ilodigwe D, Murray GD, Kassell NF, Torner J, Kerr RS, Molyneux AJ, et al. Sliding dichotomy compared with fixed dichotomization of ordinal outcome scales in subarachnoid hemorrhage trials. J Neurosurg 2013;1 18:3-12.

17. Jang $Y$, llodigwe D, Macdonald R. Metaanalysis of tirilazad mesylate in patients with aneurysmal subarachnoid hemorrhage. Neurocrit Care 2009;10:141-7.

18. Jansen K, Lagae L. Cardiac changes in epilepsy. Seizure 2010;19:455-60.

19. Jennett B, Bond M. Assessment of outcome after severe brain damage: A practical scale. Lancet 1975;305:480-4.

20. Kang J, Shen W, Macdonald R. Why does fever trigger febrile seizures? J Neurosci 2006;26:2590-7.

21. Kassell N, Haley E, Apperson-Hansen C, Alves W. Randomized, double-blind, vehicle-controlled trial of tirilazad mesylate in patients with aneurysmal subarachnoid hemorrhage: A cooperative study in Europe, Australia and New Zealand. J Neurosurg 1996;84:22I-8.

22. Kim W, Neal L, Hoh B. Risk factors, incidence and effect of cardiac failure and myocardial infarction in aneurysmal subarachnoid hemorrhage patients. Neurosurgery 2013;73:450-7.

23. Kumar A, Brown R, Dhar R, Sampson T, Derdeyn CP, Moran CJ, et al. Early vs delayed cerebral infarction after aneurysm repair after subarachnoid hemorrhage. Neurosurgery 2013;73:617-23.

24. Lannes M, Teitelbaum J, del Pilar Cortes M, Cardoso M, Angle M. Milrinone and homeostasis to treat cerebral vasospasm associated with subarachnoid hemorrhage: The Montreal Neurological Hospital protocol. Neurocrit Care 2012;16:354-62.

25. Lanzino G, Kassel N. Double-blind, randomized, vehicle-controlled study of high-dose tirilazad mesylate in women with aneurysmal subarachnoid hemorrhage. Part II. A cooperative study in North America. J Neurosurg 1999;90:1018-24.

26. Lanzino G, Kassell NF, Dorsch NW, Pasqualin A, Brandt L, Schmiedek P, et al. Double-blind, randomized, vehicle-controlled study of high-dose tirilazad mesylate in women with aneurysmal subarachnoid hemorrhage. Part I. A cooperative study in Europe, Australia, New Zealand and South Africa. J Neurosurg 1999;90:1011-7.

27. Layon A, Gabrielli A, Friedman W. Textbook of neurointensive care. Philadelphia: Saunders; 2004.

28. Lee K. The neurolCU book. New York: McGraw-Hill Medical; 2012.

29. Lee VH, Oh JK, Mulvagh SL, Wijdicks EF. Mechanisms in neurogenic stress cardiomyopathy after aneurysmal subarachnoid hemorrhage. Neurocrit Care 2006;5:243-9.

30. Lindekleiv H, Sandvei MS, Romundstad PR, Wilsgaard T, Njølstad I, Ingebrigtsen $\mathrm{T}$, et al. Joint effect of modifiable risk factors on the risk of aneurysmal subarachnoid hemorrhage: A cohort study. Stroke. 2012;43:1885-9.

31. Lipsman N, Tolentino J, Macdonald RL. Effect of country or continent of treatment on outcome after aneurysmal subarachnoid hemorrhage. J Neurosurg 2009; I I I:67-74.

32. Lo B, Fukuda H, Nishimura $\mathrm{Y}$, Wan $\mathrm{Y}$, Lo A. Brain-body interactions: Contemporary outcome prediction in aneurysmal subarachnoid hemorrhage using bayesian neural networks and fuzzy logic. New York: Nova Biomedical; 2014.

33. Lo B, Macdonald R, Baker A, Levine M. Clinical outcome prediction in aneurysmal subarachnoid hemorrhage using Bayesian neural networks with fuzzy logic inferences. Comput Math Methods Med 2013;20I3:904860. 
34. Mangieri P, Suzuki K, Ferreira M, Domingues L, Casulari LA. Evaluation of pituitary and thyroid hormones in patients with subarachnoid hemorrhage due to ruptured intracranial aneurysm. Arq Neuropsiquiatr 2003;6I:14.

35. Mazarati A. Cytokines: A link between fever and seizures. Epilepsy Curr 2005;5:169-70.

36. Mrozek S, Vardon F, Geeraerts T. Brain temperature: Physiology and pathophysiology after brain injury. Anesthesiol Res Pract 20 I 2;20 I 2:989487.

37. O'Carroll RE, Hayes PC, Ebmeier KP, Dougall N, Murray C, Best J, et al. Regional cerebral blood flow and cognitive function in patients with chronic liver disease. Lancet 199|;337:I250-3.

38. Park J, Woo H, Kang D, Kim YS, Kim MY, Shin IH, et al. Formal protocol for emergency treatment of ruptured intracranial aneurysms to reduce in-hospital rebleeding and improve clinical outcomes. J Neurosurg 2015; | 22:383-9 I.

39. Payen J, Fauvage B, Falcon D, Lavagne P. Brain oedema following blood-brain barrier disruption: Mechanisms and diagnosis. Ann Fr Anesth Reanim 2003;22:220-5.

40. Rowland L, Pedley T, Merritt H. Merritt's neurology. Philadelphia, PA: Lippincott Williams \& Wilkins; 2010.

4I. Rowland MJ, Hadjipavlou G, Kelly M, Westbrook J, Pattinson KT. Delayed cerebral ischaemia after subarachnoid haemorrhage: Looking beyond vasospasm. Br J Anaesth 20I2;109:3 I5-29.
42. Sadamasa N, Koyanagi M, Fukuda H, Chin M, Handa A, Yamagata S. Is aneurysm repair justified for the patients aged 80 or older after aneurysmal subarachnoid hemorrhage? J Neurointerv Surg 20I4;6:664.

43. Schneider H, Kreitschmann-Andermahr I, Ghigo E, Stalla GK, Agha A. Hypothalamopituitary dysfunction following traumatic brain injury and aneurysmal subarachnoid hemorrhage: A systematic review. JAMA 2007;298: 1429-38.

44. Steyerberg EW, Vickers AJ, Cook NR, Gerds T, Gonen M, Obuchowski N, et al. Assessing the performance of prediction models: A framework for traditional and novel measures. Epidemiology 20I0;2I:128-38.

45. Todd MM, Hindman BJ, Clarke WR, Torner JC, Weeks JB, Bayman EO, et al. Perioperative fever and outcome in surgical patients with aneurysmal subarachnoid hemorrhage. Neurosurgery 2009;64:897-908.

46. Torbey M. Neurocritical care. Cambridge; New York: Cambridge University Press; 2010.

47. Varelas P. Seizures in critical care: A guide to diagnosis and therapeutics. Totowa, NJ: Humana Press; 2005.

48. Wijdicks $\mathrm{E}$. The practice of emergency and critical care neurology. New Work: Oxford University Press; 2010.

49. Zhang S, Wang L, Liu M, Wu B. Tirilazad for aneurysmal subarachnoid haemorrhage. Cochrane Database Syst Rev 2010;(2):CD006778. 